\title{
Suppressing Near-Receiver Scattered Waves from Seismic Land Data
}

\author{
Xander Campman and Gérard Herman* \\ Earth Resources Laboratory \\ Dept. of Earth, Atmospheric, and Planetary Sciences \\ Massachusetts Institute of Technology \\ Cambridge, MA 02139 \\ * Shell International E \& P, Rijswijk, The Netherlands
}

\begin{abstract}
When upcoming body waves travel through a heterogeneous near-surface region, the continuity of the wavefront can be diminished by scattering. We discuss a multichannel method to predict and subtract near-receiver scattered waves, such that the continuity and trace-to-trace coherency of wavefronts increases. We apply this method to a part from a field-data set which was acquired in an area with significant near-surface scattering. We show that the method increases trace-to-trace coherency in a reflection event. Moreover, application of our method improves the results obtained from application of a dip filter only, because we remove parts of the scattered wave with apparent velocities that are typically passed by the pass-zone of the dip filter.
\end{abstract}

\section{Introduction}

The near subsurface can affect seismic data in several ways. For example, lateral velocity variations in the near subsurface and variations in layer thickness or topography cause variations in the arrival times and amplitudes of the upcoming wave front. When the dimensions of anomalies are comparable to the dominant wavelength, they can excite secondary (or scattered) waves. Moreover, spatially limited structures, like dunes, caves or underground channels, may cause resonances (Levander, 1990; Combee, 1994), while the presence of the surface and near-surface layers allow for the generation of guided wave types, like groundroll. For our discussion here, it is especially important to note that, in addition to a time shift, scattering and resonances cause time-varying phase and amplitude distortions in upcoming reflection events.

In seismic exploration, some of these unwanted effects are traditionally suppressed during acquisition using field arrays. Field arrays can greatly improve the data quality by way of stacking, which increases signalto-noise (Cooper, 2004, e.g.), while suitably chosen patterns can suppress ground roll (Morse and Hildebrandt, 1988). However, as the complexity of the overburden increases, rapid lateral variations on the scale of an array or less can cause perturbations that renders the use of field arrays less effective (Blaquière and Ongkiehong, 2000; Muyzert and Vermeer, 2004). In such cases, stacking in the field diminishes the integrity of the output trace which in turn limits the maximum obtainable resolution in the final image.

For this reason, seismic exploration has witnessed a trend towards acquiring data with smaller geophone groups up to the point where single receivers are recorded (Burger et al., 1998; Baeten et al., 2000, for example). The idea of single-receiver acquisition is to preserve as much signal as possible. The philosophy of these methods is that whatever noise was suppressed by stacking the array, can be done better 
after preprocessing as the dense sampling offers greater flexibility in processing (Baeten et al., 2001). After preprocessing, conventional groups can be formed, which are then expected to be more effective.

Time variations incurred by rapid near-surface variations are usually corrected with 'residual statics' (see Cox, 1999, for an overview). In statics, one assumes that the effect of the near subsurface is a pure time delay and that these delays are surface consistent, implying that each trace at a given surface location gets the same time delay. This is usually explained by assuming vertical ray paths through the overburden. In addition, it is assumed that the near-surface effects do not vary with reflection time. Thus, the traditional statics model assigns the same uniform time shift to each trace from a distinct surface location. In this way, a single time shift corrects the entire trace.

For the simultaneous estimation of amplitude and time anomalies, surface-consistent deconvolution methods have been developed (Cambois and Stoffa, 1993; Perkins and Calvert, 2001, e.g.). Using the principle of surface consistency, they are essentially based on the same assumptions as statics methods, resulting in single-channel corrections.

A commonly used method to remove surface waves is filtering in the wavenumber-frequency domain (Yilmaz, 2001, e.g.). This method uses the difference in apparent velocities of different events or wave types. The (apparent) velocity of surface waves is typically very low compared to the apparent velocity of upcoming reflections. In the wavenumber frequency domain, these events separate because they align along lines with different slopes. By defining a suitable pass zone in the $k-f$ domain, all events outside the pass zone are rejected and the data are subsequently transformed back to the spatial-time domain. However, because scattering takes place close to the receivers (i.e. in the near field), these surface waves do not have linear move out yet and hence they have a part with higher apparent velocities. These parts may be mapped in the pass zone in a dip filter and cause interference.

We identify two main effects that the near-surface region has on upcoming body waves: a time shift in the first breaks plus time varying phase and amplitude anomalies. In most cases, these effects have also been treated separately - as we have seen above. The time shift is the familiar 'static shift', which may be corrected by current static methods. The additional anomalies, however, are caused by the fact that the reflection event itself excites secondary waves when it hits a scatterer near the surface. Because it is excited close to the receivers (i.e. in the near-field), this scattering interferes with the reflection event, thereby diminishing the trace-to-trace coherency. Based on these observations we argue that improvements in correcting for this type of near-surface anomalies can be made with a wave-field based (multichannel) method that takes near-surface scattering into account.

In this paper, we review a wave-field-based method to estimate and suppress near-receiver scattered waves that was proposed in Herman et al. (2000) for removing scattered tubewaves from Vertical Seismic Profiling data. Campman et al. (2005) generalized this scheme for 3-D surface seismic geometries and validated it using synthetic and laboratory-scale data. In particular, we discuss the application of the algorithm

developed in Campman et al. (2005) to a part from a densely sampled field-data set, acquired in an area with substantial near-surface scattering, placing emphasis on (quantifying) the improvements in trace-to-trace coherent and continuity in reflection events. We show that our method complements the wavenumberfrequency domain filter, because it mainly suppresses the parts of the scattered waves with higher apparent velocities.

\section{Method}

The objective of our method is to get an estimate of the wave field that would have been measured in the absence of scatterers in the near-surface region. Note that the remaining wave field can still be quite 

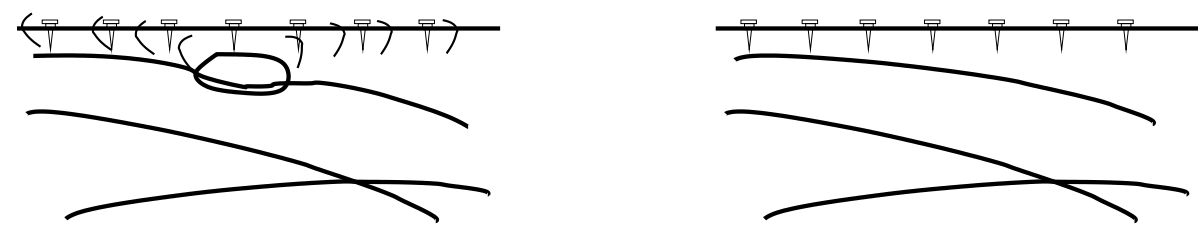

Figure 1: (a) Actual situation: upcoming body waves excite scattered surface waves when they hit a nearsurface scatterer. (b) Desired situation.

complex; it may contain primary reflections, multiples, refractions, etc. We have sketched the situation in Figure 1 where (a) depicts the actual state with scatterer and (b) represents the desired state without scattering. We use that idea with knowledge of the near-surface scattering distribution, one can model and subsequently subtract near-surface scattered waves (Blonk, 1994).

\section{Scattered noise model}

On account of linearity of the elastic wave field, the vertical velocity component $v(\underline{\mathrm{x}}, t)$, measured at position $\underline{\mathbf{x}}=(x, y, z)$ and due to a fixed vertical point source of force type can be written as

$$
v(\underline{\mathbf{x}}, t)=v^{0}(\underline{\mathbf{x}}, t)+v^{1}(\underline{\mathbf{x}}, t) .
$$

Here, $v$ is the measured field, $v^{0}$ is the field that would have been measured if the overburden were homogeneous and $v^{1}$ is the part of the wave field that accounts for scattering from heterogeneities close to the acquisition surface. Thus, we want an estimate of $v^{0}$. Our approach is to find the scattered noise, $v^{1}$, and then subtract it from the data as in Equation (11). From the elastic wave-equation for particle displacement, we can derive an approximate integral representation for the scattered noise in terms of the vertical component of particle velocity, measured at the surface $z_{0}$ :

$$
v^{1}\left(\underline{\mathbf{x}}_{l}, z_{0}, \omega\right)=\int_{\underline{\mathbf{x}}_{l}^{\prime} \in \Sigma} u^{G}\left(\underline{\mathbf{x}}_{l}-\underline{\mathbf{x}}_{l}^{\prime}, \Delta z, \omega\right) \sigma\left(\underline{\mathbf{x}}_{l}^{\prime}, z_{1}, \omega\right) v\left(\underline{\mathbf{x}}_{l}^{\prime}, z_{1}, \omega\right) \mathrm{d} \underline{\mathbf{x}}_{l}^{\prime},
$$

where $u^{G}$ is the vertical component of the Green's displacement tensor, due to a vertical point force. Horizontal position is denoted by $\underline{\mathrm{x}}_{l}=(x, y), z_{1}$ is the scattering depth and $\Delta z=z_{0}-z_{1}$. The impedance distribution is denoted by $\sigma ; \omega$ is angular frequency. The surface $\Sigma$ is the area occupied by the receivers (i.e. the acquisition surface). If the scattering takes place close to the surface $\left(z_{1} \approx z_{0}\right)$, we can approximate the field at $z_{1}$ by the field recorded field at depth $z_{0}$. Note that the integral is over a surface and we thus express scattering by a scattering volume in terms of a surface impedance distribution. The validity

of this assumption for scattering of surface waves close to the surface is investigated in Blonk (1994). To account for variations in the actual depth of the scatterers we allow the impedance distribution to depend on frequency. Equation (2) allows us to calculate the scattered field directly, once the scattering distribution $\sigma$ is known. In the next section, we describe a sequence of steps that leads to an independent estimate of $\sigma$, form the data itself.

\section{Inverse scattering}

Suppose the data contain many reflections from deeper layers. All these events excite surface waves at the same heterogeneities close to the acquisition surface. This implies that we can use the scattered energy from a single event to estimate the impedance distribution and use it to predict the scattered energy on every 
reflection using Equation (2). In fact, this is comparable to residual statics methods, where one selects a time window around a strong reflection event from the data, to derive the time-shifts for each trace separately. These independently obtained time shifts are then applied to the entire trace. Instead of this single-channel operation, we now select one event to derive an impedance distribution to estimate the scattered energy (i.e. a multi-channel operation).

First, we select an event:

$$
v(\underline{\mathbf{x}}, t)=d(\underline{\mathrm{x}}, t)+r(\underline{\mathrm{x}}, t),
$$

where $v$ are the data, $d$ is the selected event and $r$ denotes the rest of the data. Selecting $d$ can be done by time windowing. The window should be long enough to include scattering tails but it should not include other events. Next, we decompose the strong event $d(\underline{\mathbf{x}}, t)$, in a similar way as in Equation (1):

$$
d(\underline{\mathbf{x}}, t)=d^{0}(\underline{\mathbf{x}}, t)+d^{1}(\underline{\mathbf{x}}, t) .
$$

Here, $d^{0}$ is the field in the near surface that would exist without scattering and $d^{1}$ is the scattered field, excited by the incident field being scattered from heterogeneities in the near surface. The scattered energy can be obtained via spatial filtering. This is discussed in more detail in the following examples. The impedance distribution is determined by minimizing an $L^{2}$-norm, using a conjugate-gradient method Van den Berg, 2002, for example). To set up the minimization scheme, we write Equation (2) for a single event, in the form

$$
d^{1}=\mathcal{K} \sigma,
$$

where $\sigma$ is the surface impedance distribution and the operator $\mathcal{K}$ is defined as

$$
\{\mathcal{K} \sigma\}\left(\underline{\mathbf{x}}_{l}, z_{0}, \omega\right)=\int_{\underline{\mathbf{x}}_{l}^{\prime} \in \Sigma} u^{G}\left(\underline{\mathbf{x}}_{l}-\underline{\mathbf{x}}_{l}^{\prime}, \Delta z, \omega\right) \sigma\left(\underline{\mathbf{x}}_{l}^{\prime}, z_{1}, \omega\right) d\left(\underline{\mathbf{x}}_{l}^{\prime}, z_{1}, \omega\right) \mathrm{d} \underline{\mathbf{x}}_{l}^{\prime} .
$$

We then minimize the squared difference between the observed scattered field and the reconstructed scattered field, regularized by the norm of the distribution of scatterers:

$$
F=\frac{\left\|d^{1}-\mathcal{K} \sigma\right\|^{2}}{\left\|d^{1}\right\|^{2}}+\lambda\|\sigma\|^{2}
$$

where the size of $\lambda$ determines the penalty on the norm of the distribution of scatterers. By assumption, the scatterers are close to the surface so that in Equation [6, we can substitute the field at depth $z_{1}$ with the field at $z_{0}$, leaving $\sigma$ the only unknown. In contrast to Born-type imaging methods, this method accounts for multiply scattered waves.

\section{Schlumberger Michigan Experiment: Glacial Till}

We describe the application of our algorithm to parts from a field-data set. These data have been provided by Schlumberger Cambridge Research/WesternGeco. It was collected at a field experiment in Michigan (US) and it comprises two densely sampled receiver lines recorded in an area with a significant amount of near-surface heterogeneity. 


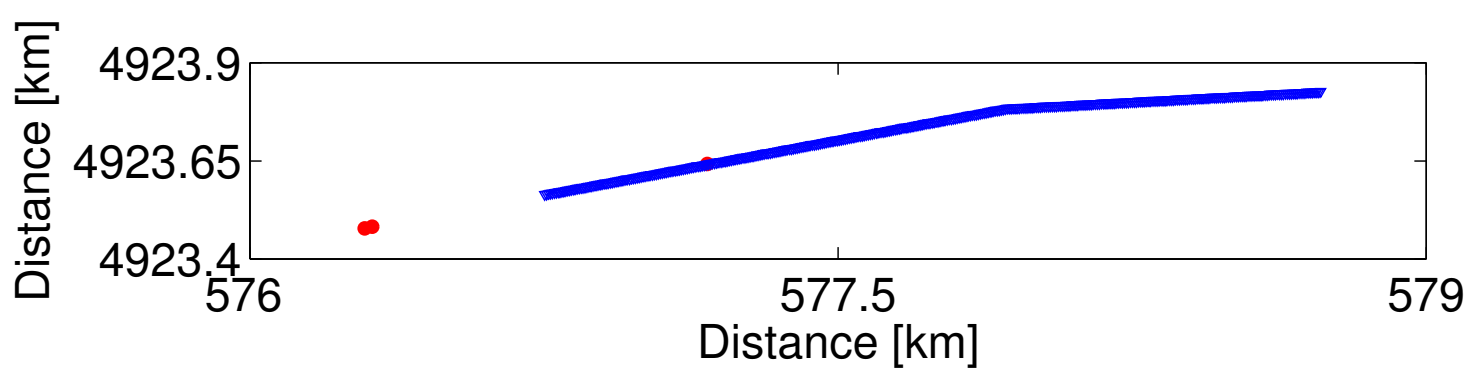

Figure 2: Geometry of one line from the Michigan experiment.

\section{Geometry}

During the summer of 2002, Schlumberger/WesternGeco recorded two lines with 401 multi-component receivers each, in a cross-spread geometry. From these two lines, we have analyzed the vertical component of the velocity of one line running roughly from West to East. The geometry of this line is shown in Figure 2 After analyzing these data, we have focused on the data from shot 103 and 341 in more detail (depicted in this Figure). From these shots, we have selected areas where we have applied our prediction-and-subtraction algorithm.

The receivers were fixed on the line shown in Figure 2 The total length of the receiver line is 2000 $\mathrm{m}$. Each shot is recorded by the entire line of 401 receivers with a spacing of $5 \mathrm{~m}$. For the survey, (single) explosive sources with a spacing of $10 \mathrm{~m}$ were used. No arrays were formed in the field, so that each trace from this densely sampled data set was stored on tape. Fig. 3] shows a part of shot record 103 (approximately indicated in Fig. 2).

\section{Near-surface scattering}

The data (part of which is shown in Figure 3) were collected in an area where substantial near-surface scattering is present. The near-surface region consists of a glacial till containing large boulders. Since receiver intervals (or geophone spacings) are small $(5 \mathrm{~m})$, we can clearly identify near-surface scattering. Because the near surface varies considerably along the line, we have selected a few areas where the near surface is (hopefully) not changing too much.

The arrows in Figure 3 point to some characteristic features of near-surface scattering. We observe diffraction hyperbolas marked with (1), (2), in the figure. We also note parts of diffractions with linear move-out (3),4). Note that the scattering features are consistently repeated in the later (reflection) event. This is typical of near-receiver scattering because each event passes through the same anomaly close to the receiver. For this reason, we may also call this type of noise multiplicative noise. Along the part of the line shown here, there are no significant elevation variations. Around $175 \mathrm{~m}$, a strong discontinuity is present in both the first arriving wave and the reflection (5). From looking at the elevations in the headers of the data, this discontinuity has no apparent surface manifestation. This observation leads us to believe that the discontinuity is also caused by near-surface scattering.

Figure 3 b shows part of the wavenumber-frequency $(k-f)$ spectrum of the data shown in Figure 3 . The velocity of the first arriving wave is roughly $1625 \mathrm{~ms}^{-1}$ (indicated by the dashed line in Fig 3 $\mathrm{k}$ ). Because this wave has linear move-out we should be able to observe a coherent alignment in the $k-f$ spectrum with a slope of about $.0007 \mathrm{sm}^{-1}$. The beginning of this line is indicated with the dashed line in Figure $3 \mathrm{~b}$. 


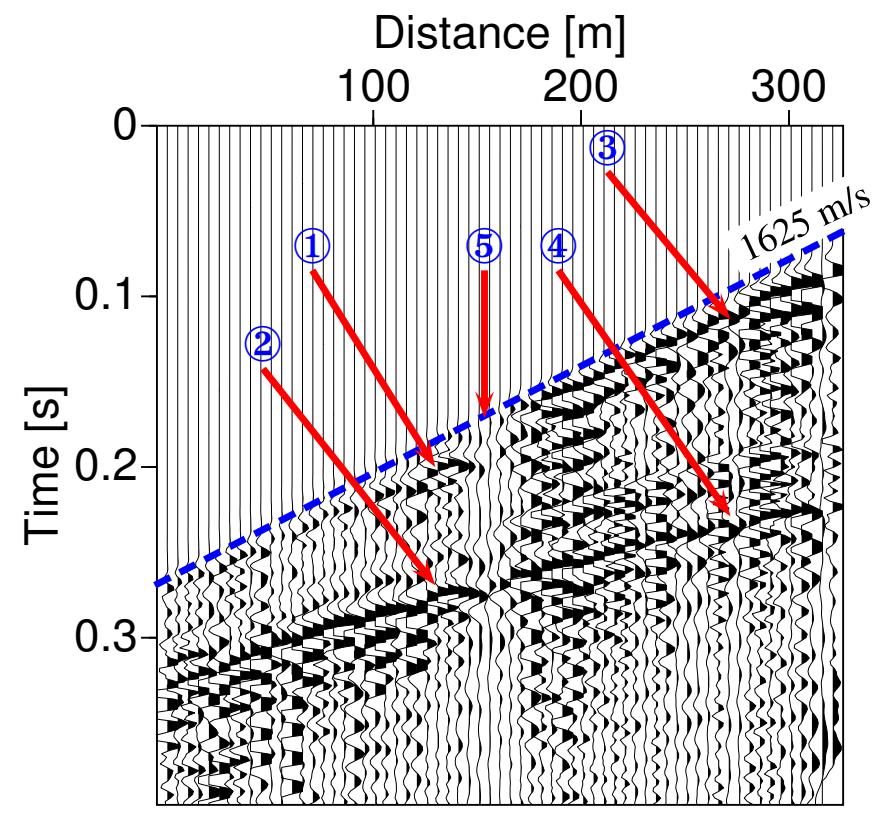

(a)

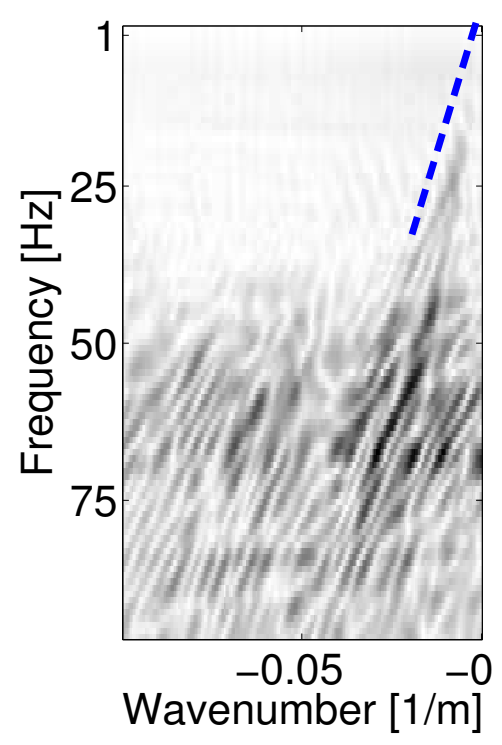

(b)

Figure 3: (a) Detail of shot record 103. We clearly observe the effects of the near subsurface on the two events in this display. One can identify diffraction hyperbolas around $125 \mathrm{~m}$ and strong interference around $150 \mathrm{~m}$, typical of near-surface scattering. They are indicated by the arrows; the numbers are explained in the text. (b) Wavenumber-frequency domain spectrum of the data displayed in (a). In this domain, we also observe effects introduced by the near subsurface. These are explained in the text.

We observe that due to near-surface perturbations, this event is not a well-defined linear alignment as we would expect to get from a linear arrival in the data. Moreover, we observe that much energy is 'smeared'

over the $k-f$ space due to amplitude and arrival time anomalies (Panea et al., 2003). We observe dipping lines, almost parallel to the dashed line, typical of random static effects (Blaquière and Ongkiehong, 2000; Panea et al., 2003). This noise is dispersed throughout the $k-f$ domain and is thus partly localized in the pass band of a dip filter (in the context of the effect of perturbations on linear noise arrivals, this is discussed in Cox, 1999, pp. 311-313). This effect is similar for scattering hyperbolas induced by nearsurface scattering, as the apex has higher apparent velocities than linear coherent noise.

\subsubsection{Wave-Field Separation}

Wave-field separation is part of the preconditioning of the data before inversion. To estimate a near-surface impedance function, we require an estimate of the near-surface scattered field. Because we assume that the scattering takes place immediately under the receivers, we can assume that each event passes through the same heterogeneous domain. Consequently, we need only one event to characterize the immediate subsurface. This is the 'reference' event.

In Figure 3 , such an event is the first arriving wave. To select the reference event, we place a time- 
window around it. In this case we use a constant velocity (from the data we estimate $c \approx 1625 \mathrm{~ms}^{-1}$ ):

$$
\begin{aligned}
t_{\min }(x) & =T_{0}-x / c-\mu T_{w}, \\
t_{\max }(x) & =T_{0}-x / c+(1-\mu) T_{w} .
\end{aligned}
$$

Here, $x$ denotes the offset, $c$ the velocity, $T_{0}$ the intercept time of this event (in this case about $0.1 \mathrm{~s}$ ) and $T_{w}$ is the length of the time window. The window length $T_{w}$ should be long enough to include scattering coda, but, preferably not too long so that it includes other events. The parameter $\mu$ controls the portion of this window to include the reference event and the portion to include scattering following this event so that it does not have to be centered around $T_{0}$ (if $\mu=0.5$, the window is centered around $T_{0}$ ). Defined in this way, $t_{\min }(x)$ described the minimum time selected for each trace, while $t_{\max }(x)$ describes the maximum time included for each trace. In practice, we also taper the edges of this window to reduce artifacts in further processing.

The next step is to separate the wave field in the embedding from the wave field due to local perturbations. Because scattering takes place close to the receivers (i.e. in the near field), it interferes with the incident wave. For this reason, careful processing is required. Various methods can be used to separate the local perturbations from this event. For example, Campman et al. (2005) use a wavenumber-frequency domain filter for a (approximately) plane incident waves in a medium with a homogeneous near-surface region. For more complicated arrivals, for example in the presence of topography one can use smoothing techniques that afford slow variations in the incident wave field (Gersztenkorn and Scales, 1988, e.g.). In the case where different events interfere, one can use more advanced techniques, for example based on the singular value decomposition, used in vertical seismic profile processing (Mars et al., 1999, e.g.).

From looking at the data we infer that the first arriving wave varies somewhat smoothly. From this observation we presume that the embedding changes slowly. Although we have assumed that the near-surface region in our scattered-noise model is homogeneous, we can allow for some lateral variation as long as we can obtain a reasonable estimate for the wave field in the embedding through preprocessing. To afford such slow variations while separating the faster variations, we have chosen an $\alpha$-trimmed mean $(\alpha$-TM) filter (Bednar and Watt, 1984, e.g.). The window length of the $\alpha$-TM filter offers a handle on the smoothness of the changes that we want to maintain in the incident field. First, we apply linear move-out correction (LMO) to the reference event to align it horizontally. Then we apply the $\alpha$-TM filter to estimate the wave field in the embedding medium. Once we have obtained an estimate of the incident field, we subtract it from the total field in the following way (Equation (1):

$$
d^{1}(\underline{\mathbf{x}}, t)=d(\underline{\mathbf{x}}, t)-\beta d^{0}(\underline{\mathbf{x}}, t),
$$

to obtain the near-surface scattered energy $d^{1}$ for the reference event. In the subtraction we include a simple scaling factor $(\beta)$ so that the incident wave is optimally removed from the total field.

This procedure is depicted in Figures $4 \mathrm{a}-\mathrm{c}$, where the (spatial) window length for the $\alpha$-TM filter is 11 points and $\alpha=.2$. The reference event is shown in Figure 4h, while the estimated incident wave is shown in Figure $4 \mathrm{~b}$. Finally, the estimated scattered field is shown in Figure 4t. Inspection of this figure reveals that most of the smooth event has been removed from the reference event. In particular, note that the apex of the hyperbola around $130 \mathrm{~m}$ is unimpaired, which indicates that we chose the filter parameters correctly.

\subsubsection{Imaging}

Once we have an estimate of the near-surface scattered energy, the next step is to image it. This yields the near-surface impedance function. 


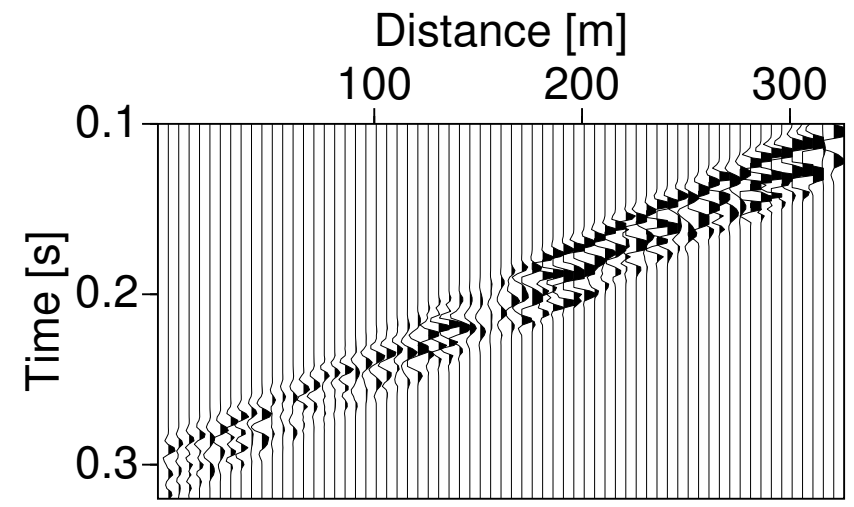

(a)

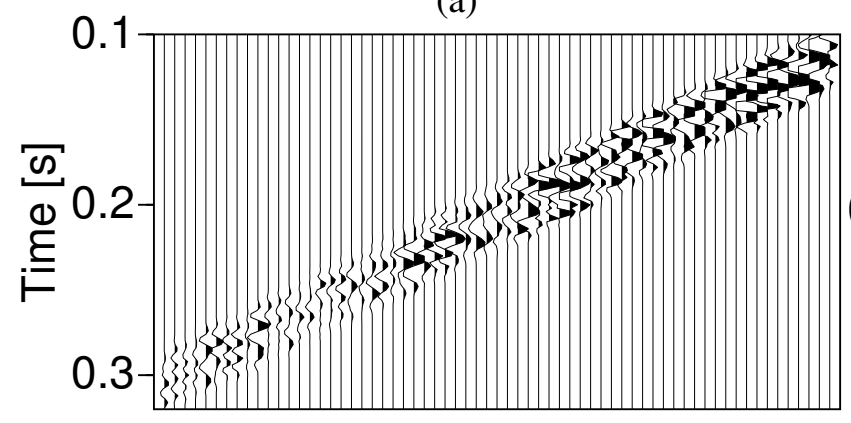

(c)

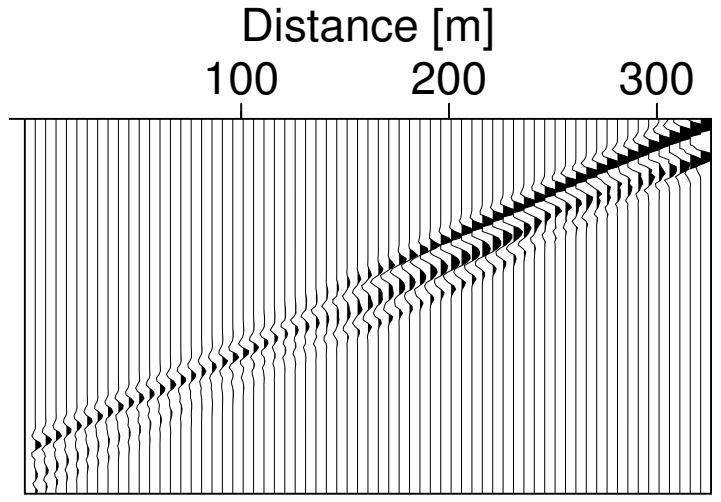

(b)

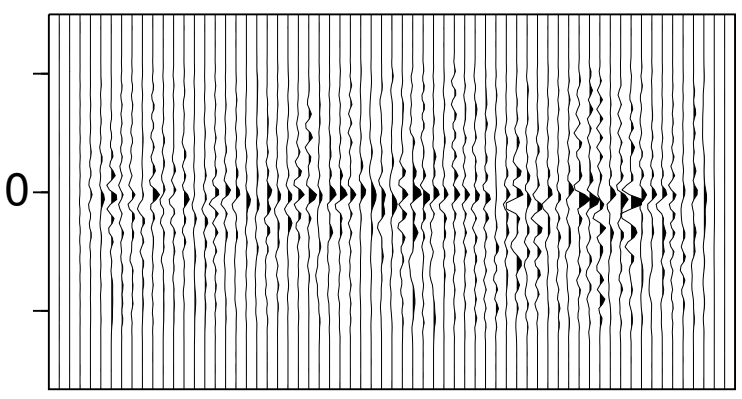

(d)

Figure 4: (a) The reference event selected from the detail of shot record 103. (b) Estimate of the incident wave field. This estimate is obtained using the $\alpha$-TM filter. (c) Estimated near-surface scattered energy. This is the wave field attributed to the presence of local heterogeneity. (d) Near-surface impedance function.

The inversion scheme is formulated in terms of minimizing a misfit between the estimated scattered field and a forward-modeled scattered field by iteratively updating the impedance function using a conjugategradient method.

The algorithm has been set up to handle 3-D data, and up to now we have used 3-D data exclusively. However, without major modifications, we can use our algorithm for 2-D data. In fact, because the data were collected along a line, and because we assume that the scattering takes place directly under the receivers, the only modification is that we now constrain the impedance function to lie on the receiver line, instead of in a surface. Apart from this, the entire inversion is carried out in the same way as in the 3-D case.

For the inversion we require a velocity model of the embedding. We use the fundamental mode Rayleighwave velocity in this area to characterize the model. We estimate this velocity from the data (the data shown here however, are selected such that there is no intefrenece form the ground roll); it is given by $c_{R} \approx 300$ $\mathrm{ms}^{-1}$. Even though the Rayleigh wave is dispersive, we may use the velocity of the fundamental mode, because our algorithm is not very sensitive to this velocity.

After minimizing the difference between the modeled scattered field and the scattered field obtained from the reference event, we obtain the impedance function shown in Figure 4t. The scattered energy has been focused around $t=0$, which justifies our assumption that the scattering takes place close to the surface. 


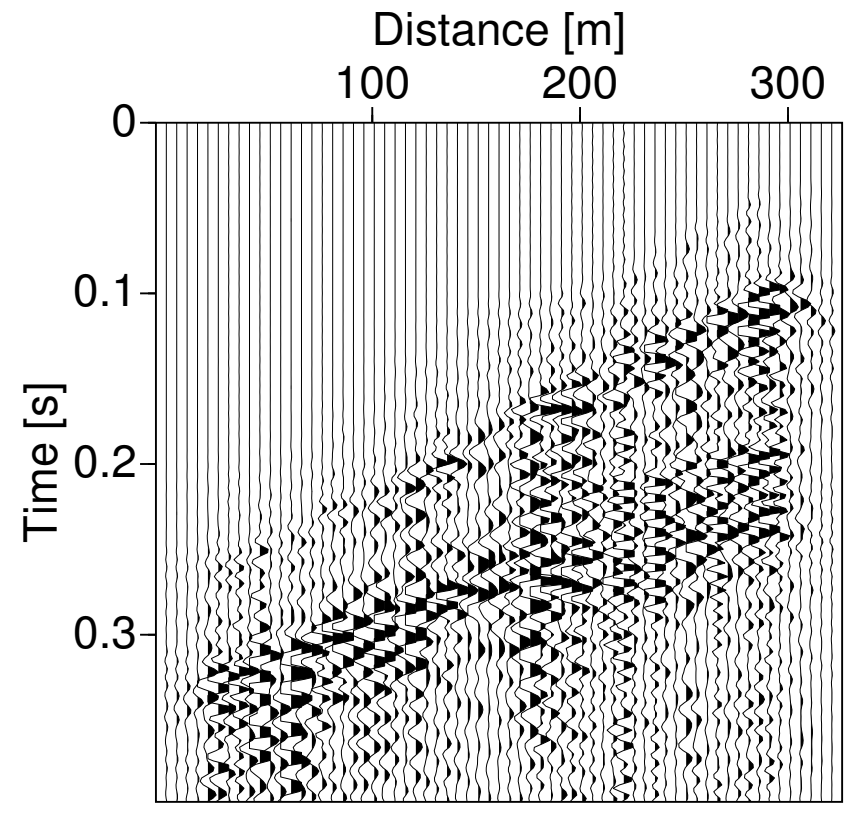

(a)

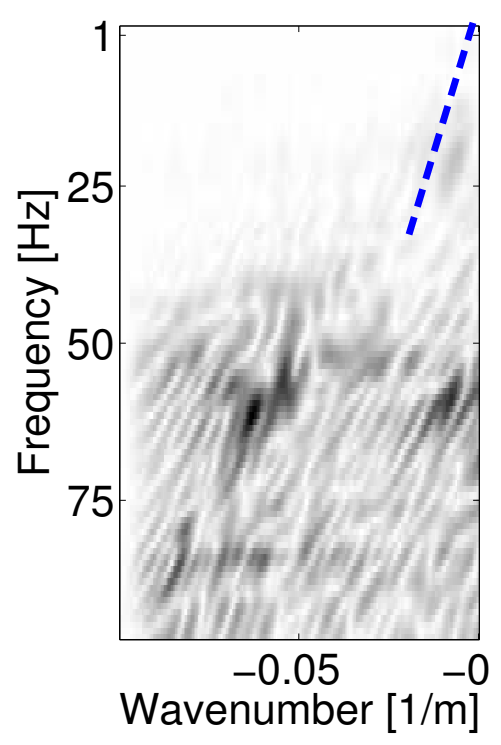

(b)

Figure 5: (a) Scattered field predicted with Equation (2) and using the impedance function of Figure $4 \mathrm{~d}$. We observe that we have mainly predicted scattered energy because there is hardly any coherent incident or reflection energy in this prediction. (b) Wavenumber-frequency domain spectrum of the predicted scattered field.

\subsubsection{Predicting and Subtracting Near-Receiver Scattered Waves}

With this estimate of the impedance function, we calculate the scattered field on the data shown in Figure 3 , using Equation (2). This predicted scattered field is shown in Figure 5. From inspecting these predicted data we conclude that we have not modeled coherent incident or reflection events, but only the part of the wave field that we attribute to the presence of near-surface heterogeneity. From this observation we conclude that our scattering model (Equation (2) ) effectively discriminates near-surface scattering from reflection events.

This can also be observed in Figure 5b, which shows the $k-f$ representation of the predicted data (the gray scales of Figure $3 \mathrm{~b}$ and $5 \mathrm{~b}$ are the same). The dashed line shows the start of the expected linear alignment of coherent energy from the first arriving wave.

Finally, we show the data after subtracting the predicted scattered energy in Figure 6 This figure demonstrates that we have removed most of the interference caused by scattering. However, there are some remnants of coherent energy with the steeper dips (for example between 75 and $125 \mathrm{~m}$, around $0.3 \mathrm{~s}$ ). This energy is most likely introduced by the ringing in the impedance function, because they appear to have a strong dominant frequency.

As a quick way of quality control, we can compare the estimate of the incident field from the reference event (Figure 4b) with the same event in Figure 6 6 . A quick glance at both figures confirms that they resemble quite well. The difference is due to the fact that we allow a small residual after the minimization, because our scattering model does not explain all the energy separated from the reference event, as it also includes incoherent noise for example. 


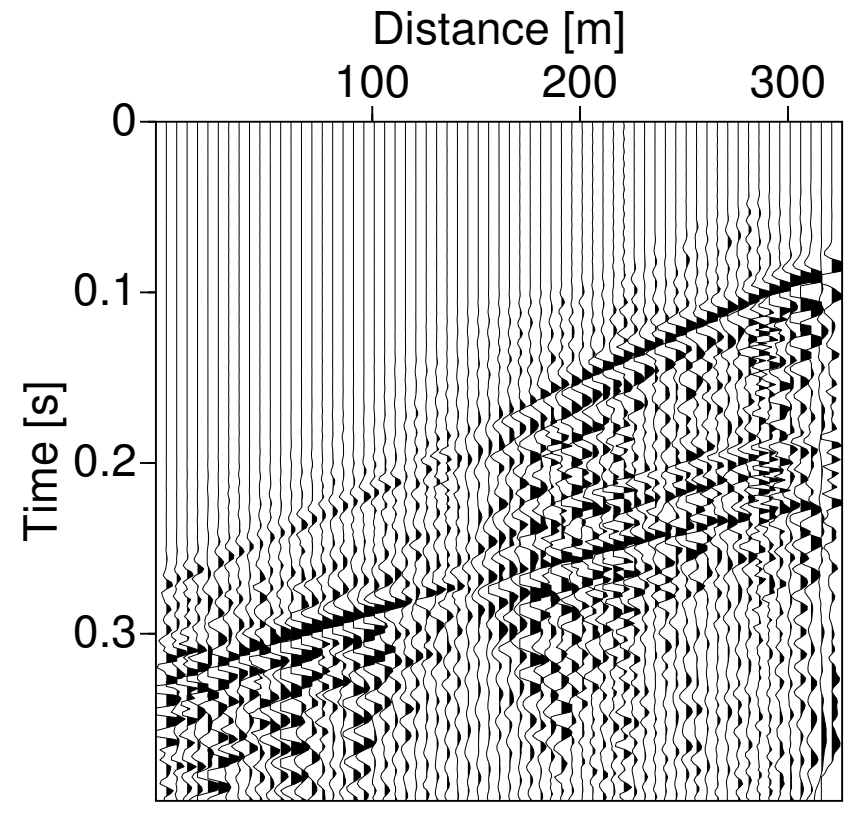

(a)

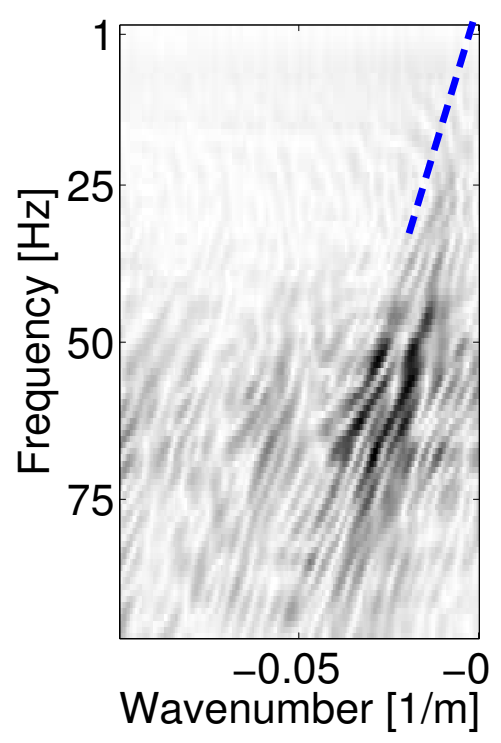

(b)

Figure 6: (a) Detail of the shot record after predicting and subtracting the near-receiver scattered waves. It corresponds to the difference of Figure $3 \mathrm{a}$ and Figure $5 \mathrm{a}$. (b) The clean data in the $k-f$ domain.

In Figure 7a, we show the input data after applying a dip filter to remove that part of the scattering that has lower apparent velocities than those in the area between the dashed line and the vertical axes. From this figure it is clear that the apexes (i.e., part of the scattered waves with higher apparent velocities) and the interference are left unaffected by the dip-filter.

After modeling and subtracting the scattered field on the record in Figure 3a, we obtain the desired output shown in Figure $7 \mathrm{~b}$ followed by the same dip filter. This is an estimate of the record that would have been measured without near-receiver scattering.

Figure 7 depicts the $k-f$ spectrum of the data shown in Figure 7. This figure shows that the dip filter removed the part of the data with lower apparent velocities, while it passed all energy in a cone around the vertical (only half of this cone is shown in this figure because the events all dip in negative direction). Figure $7 \mathrm{~d}$ shows the spectrum of the data in Figure $7 \mathrm{~b}$. The difference of these spectra is shown in Figure $7 \mathrm{~b}$. This figure shows the $k-f$ spectrum of the noise that is passed by the dip filter, but is removed by our method. This figure illustrates how our method complements the well-known dip filter.

\section{Continuity and Coherency}

(Cox, 1999, p.(p.) $451 \mathrm{f}(\mathrm{f})$ ) gives a list of quality control (QC) tools that can be used for residual statics estimations. One of the tools is to look at the continuity and trace-to-trace coherency in a common-midpoint (CMP) gather. Furthermore, (Cox, 1999, p. 457) notes that residual statics should improve the stack response of a stack made in any record domain. In principle, we should obtain similar results with our method, although we leave smooth variations intact as far as they are included as a priory information in the estimation of the incidence field of a reference event. Hence, we do not expect to get a perfectly aligned shot-gather. 


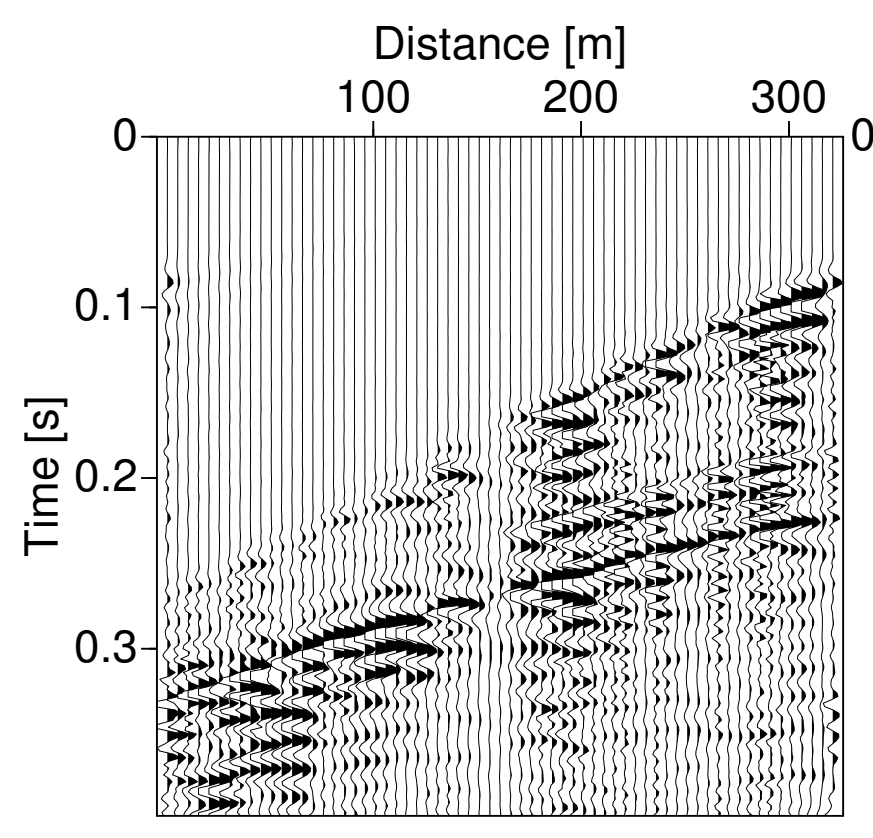

(a)

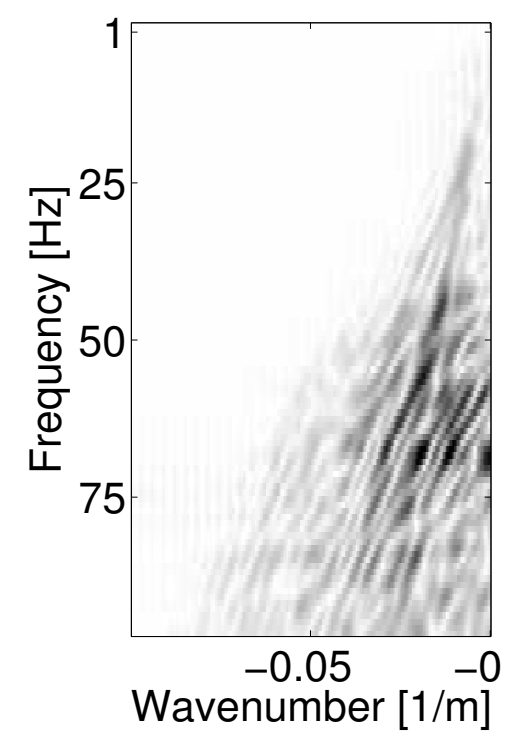

(c)

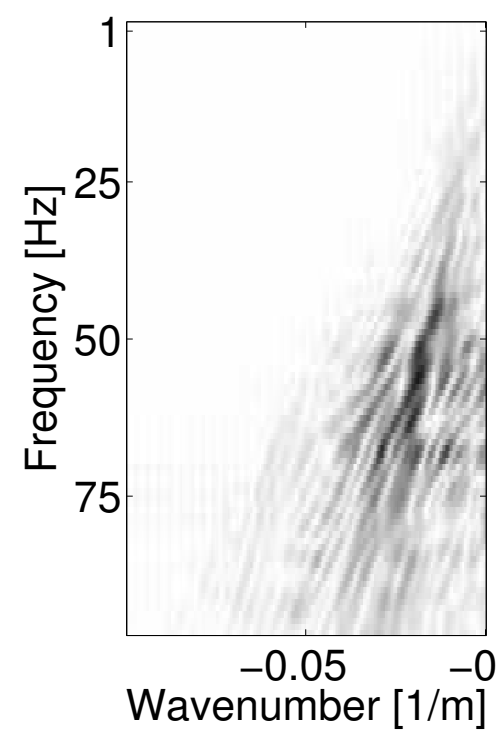

(d)

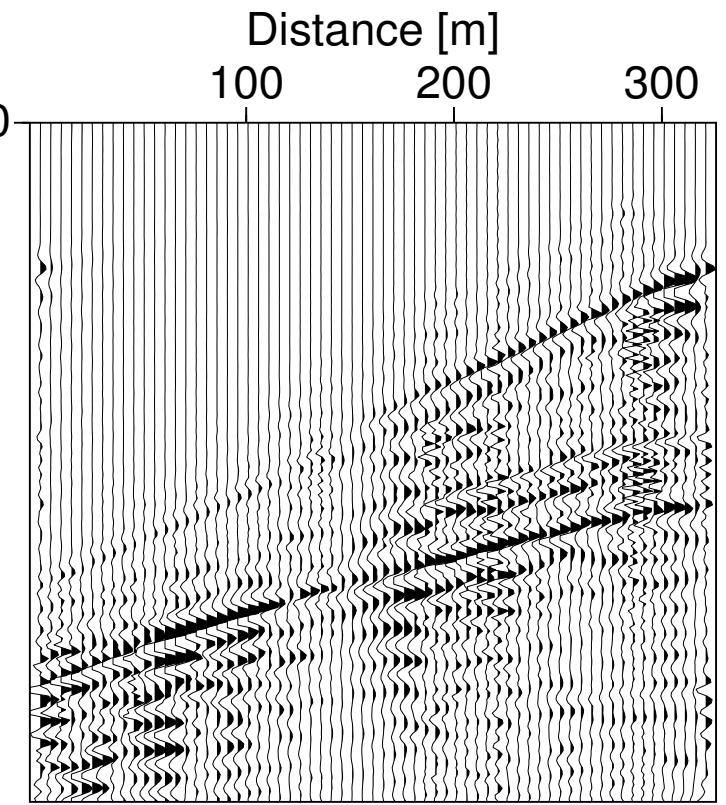

(b)

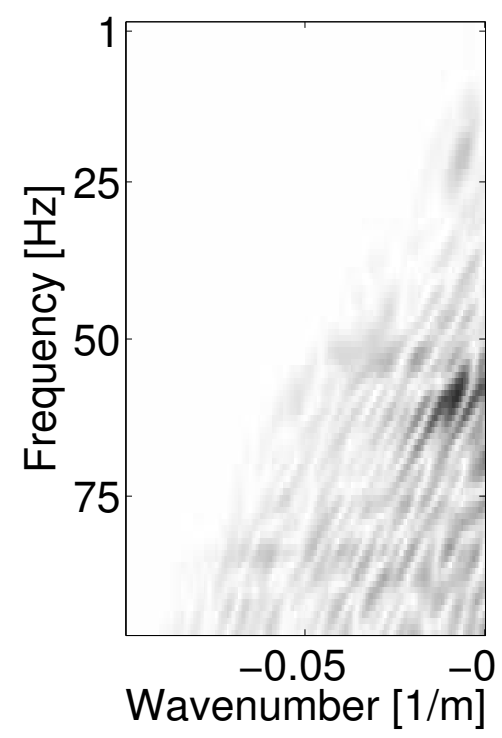

(e)

Figure 7: (a) Detail of the shot record after applying a $k-f$ filter to remove part of the record with lower apparent velocities. (b) Same detail, but after predicting and subtracting scattered waves followed by the same dip filter. Observe that we have successfully removed most of the interference. (c) Wavenumberfrequency domain spectrum of the data shown in (a). Note that the dip filter removed the part with lower apparent velocities (compare with Figure 3b). (d) Wavenumber-frequency domain spectrum of the the data shown in (b). (e) Difference between (c) and (d). This is the $k-f$ spectrum of the noise that is passed by the dip filter, but is removed by our method. 

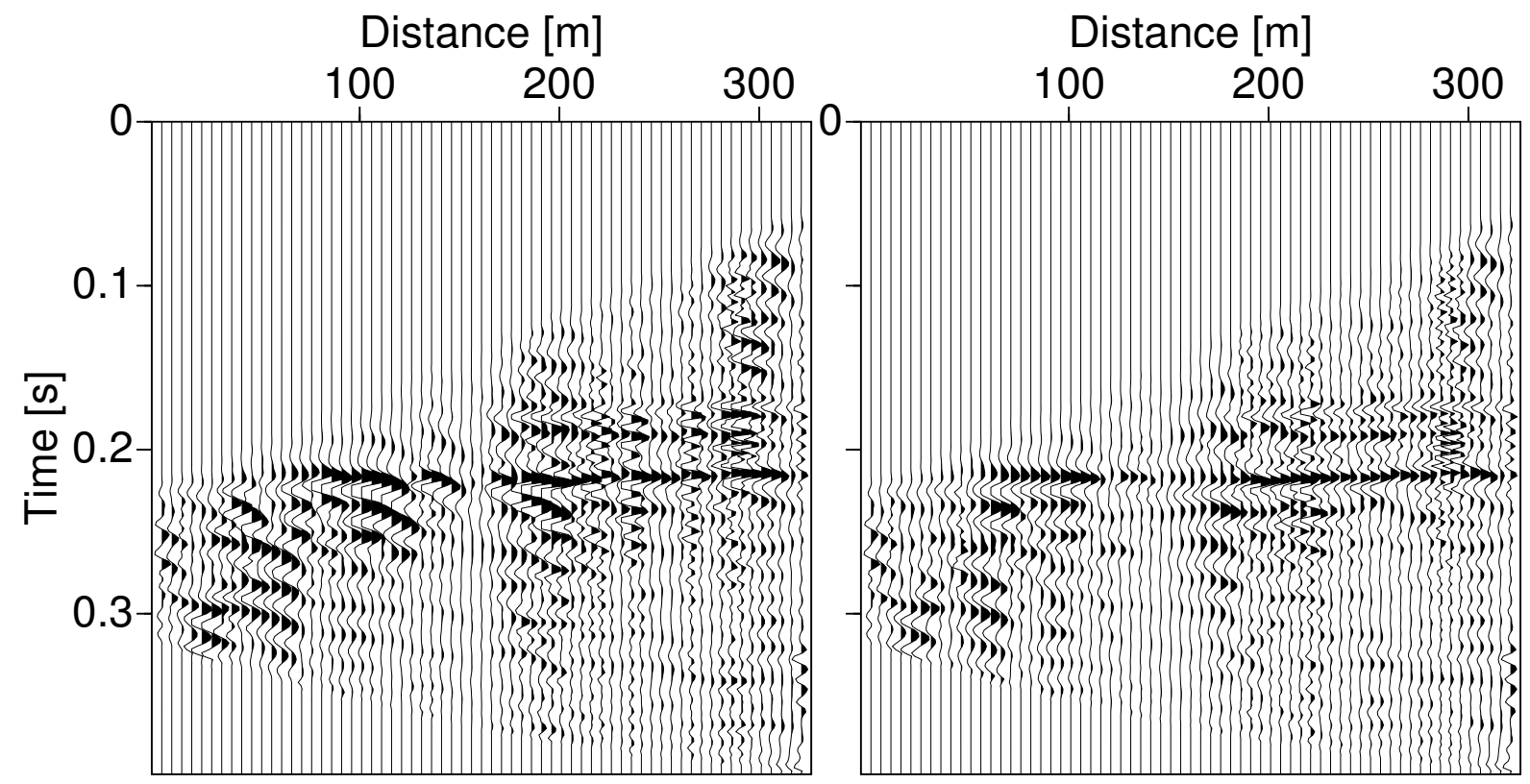

Figure 8: (a) Same as Figure 7, but after normal move-out correction. (d) Same as Figure 7b), but after normal move-out correction. This figure emphasizes the visual improvement obtained for the second event in these data.

However, compared to the initial shot gather, we should see an improvement in continuity due to the fact that we have removed the interference between the upcoming reflection and the scattered waves.

Because we have worked on common-shot records only, we use this for QC. Note, that this stack can include structural components when the near subsurface is complex. However, we assume that this structural component is negligible because we use only a portion of the data which covers a limited part of the line. From Figure 7 $\mathrm{a}-\mathrm{b}$, we see a clear visual improvement of the reflection event after we have applied our method. In Figure 8 we have applied NMO to align the reflection event. This emphasizes the improvement in continuity of this event. While it also shows that it has not been flattened completely, the variations are much less rapid than in Figure 8, implying that current statics methods may be expected to be more effective after application of our method. At the same time, we expect improved trace-to-trace coherency, because the multi-channel filter also corrects for interference that causes amplitude and phase anomalies other than shifts in the arrival times due to the difference in velocity between the scatterer and the embedding medium (like the interference in Figure 3 between 150 and $175 \mathrm{~m}$ ). To this end we calculate the semblance Telford et al. (1990). Because the method tries to restore the original wave shape after it has been altered by scattering the event will be more coherent and should produce a greater amplitude in the stack than stacking its less coherent counterpart Telford et al. (1990). Investigating the effect on the stack therefore provides a validation of our method. First, we investigate what is the effect of our method on several traces, to see if the trace-to-trace coherency has increased.

To this end we use semblance Neidell and Taner (1971). Telford et al. (1990) define semblance as the ratio of the energy of the stack compared to the energies of the individual traces over a certain time interval. 
Table 1: Semblance calculated for different events. In these tables, $R$ denotes the raw event after NMO, $R D$ denotes the event after the dip filter followed by NMO, $C$ denotes the event after subtracting the predicted scattered energy followed by NMO and $C D$ denotes the event after prediction and subtraction, followed by a dip filter and NMO. The window for the calculation of the semblance is centered around time sample $k$ and is about the length of one cycle of the waveform. The traces included run from $m_{1}$ to $m_{2}$, so that $M=m_{2}-m_{1}$. In this case we have chosen $k=115$ which corresponds to $t=0.23 \mathrm{~s}$.

\begin{tabular}{|l|l|}
\hline \multicolumn{2}{|l|}{$k=115, m_{1}=5, m_{2}=60$} \\
\hline Event & Semblance \\
$R$ & 0.21 \\
$R D$ & 0.30 \\
$C$ & 0.47 \\
$C D$ & 0.63 \\
\hline
\end{tabular}

This ratio is expressed as:

$$
S=\frac{\sum_{j=k-N / 2}^{k+N / 2}\left\{\sum_{i=1}^{M} d(j, i)\right\}^{2}}{M \sum_{j=k-N / 2}^{k+N / 2} \sum_{i=1}^{M} d^{2}(j, i)} .
$$

Here, the term in the brackets in the numerator is the sum of $M$ traces (the stack). The time gate is a window of length $N$ centered around time $k$.

Before we calculate the semblance we first apply (hyperbolic) move-out and then place a window around the event such that the remnants of the surface wave with linear move-out are not included in the calculation. Typically, the time gate for calculating the semblance is about the length of one period of the waveform. When we do this for the events shown in Figure $8 \mathrm{a}-\mathrm{b}$, before and after applying a dip filter and/or our method. We assume that there is no structural component, but, nonetheless, we don not include the farther offsets because the waveforms there are stretched due to the NMO correction. The results of these calculations are summarized in Table 1. From this table we conclude that the dip filter increases the coherency moderately, while application of our method (without a dip filter) about doubles the semblance coefficient. Finally, after we applied our method and a dip filter the semblance coefficient has increased considerably (three times the semblance coefficient of the original event).

\section{Discussion and Conclusions}

We have presented a method to predict and subtract near-receiver scattered waves from seismic land data. The objective of our method is to estimate the wavefield that would have been measured if there were no near-receiver heterogeneities. In this report we have focused on the application of this method to a densely sampled field-data set acquired in an area with significant amounts of near-surface scattering. We have quantified the trace-to-trace coherency of an upcoming reflection event before and after the application of our method. We conclude that it has significantly improved after we applied our method. In addition, we have compared the data after the application of our method to results after application of a dip filter in the wavenumber-frequency domain. This shows that our method mainly suppresses parts of the scattered waves 
with high apparent velocities that typically overlap target events in the $k-f$-domain, which are passed by the pass zone of the dip filter. In this respect, our method complements the dip filter.

In the past decade or so, wave-field-based datuming techniques have been developed to extrapolate the recorded wave field to a new 'datum' just under the overburden, thereby compensating for near-surface variations - or replacing the near-surface by a constant velocity layer (McMechan and Chen, 1990; Berryhill, 1984; Reshef, 1991). Because these methods use ray theoretical operators to redatum the data, they require an accurate velocity model of near subsurface. In complex near-surface regions, this is a serious drawback, because an accurate velocity model is difficult to obtain. For this reason, 'data-driven' redatuming techniques, like those based on Common Focus Point technology (Hindriks and Verschuur, 2001; Kelamis et al., 2002) may be preferable in areas with strong heterogeneity. However, in these areas, rapid changes in topography or layers may cause scattering and resonances. These are phenomena that are not modeled by ray theoretical methods like the redatuming techniques and, thus, the data have to be conditioned before application of such schemes. Our method however, is aimed at removing rapid variations and is based on a scattering formulation. Hence, our method also complements redatuming methods. One can fully benefit from redatuming methods in complex overburden when our method is applied first.

We are currently testing the estimation of the impedance distribution from a few events to get an impedance function consistent with a few shots, making use of the fact that the impedance function is surface consistent (Campman and Herman, 2005).

\section{Acknowledgments}

This work was carried out at Delft University and was supported by the Dutch Technology Foundation (STW). We thank Schlumberger Cambridge Research (SCR), WesternGeco and Saudi Aramco (SA) for providing the field data and giving permission to show the results. We thank Everhard Muyzert (SCR) for his efforts in providing these data and for discussions and Remco Romijn (Delphi, Delft University) for his helpful advice during processing. Xander Campman acknowledges support from Shell GameChanger.

\section{References}

Baeten, G. J. M., Belougne, V., Combee, L., Kragh, E., Laake, A., Martin, J. E., Orban, J., Özbek, A., and Vermeer, P. L. (2000). Acquisition and processing of point receiver measurements in land seismic. In Exp. Abstr., 70th Ann. Internat. Mtg., pages 41-44. Soc. of Expl. Geoph.

Baeten, G. J. M., Combee, L., and West, L. (2001). Static perturbation corrections on single sensor data. In Ext. Abstr., 63rd Mtg., pages IS-01. Europ. Assn. Geophys. Eng.

Bednar, J. B. and Watt, T. L. (1984). Alpha-trimmed means and their relationship to median filtering. IEEE Trans. on Acoust., Speech, Signal processing., 32:145-153.

Berryhill, J. R. (1984). Wave-equation datuming before stack. Geophysics, 49:2064-2066.

Blaquière, G. and Ongkiehong, L. (2000). Single sensor recording: anti-alias filtering, perturbationsand dynamic range. In Exp. Abstr., 70th Ann. Internat. Mtg., pages 29-32. Soc. of Expl. Geoph.

Blonk, B. (1994). Removal of scattered surface waves from seismic data. PhD-thesis, Delft University. 
Burger, P., Garotta, R., and Granger, P.-Y. (1998). Improving resolution and seismic quality assurance through field preprocessing. The Leading Edge, 17:1562-1568.

Cambois, G. and Stoffa, P. (1993). Surface-consistent deconvolution in the log/fourier domain. Geophysics, 57:1099-1111.

Campman, X. and Herman, G. (2005). Multichannel, surface-consistent, near-surface corrections for densely ampled land data. In Workshop: Advances and Challenges in Near Surface Seismic, 66th Mtg. Europ. Assn. Geophys. Eng.

Campman, X. H., van Wijk, K., Scales, J. A., and Herman, G. C. (2005). Imaging and suppressing nearreceiver scattered surface waves. Geophysics, 70:V21-V29.

Combee, L. (1994). Wavefield scattering by a 2-d near-surface elliptic anomaly. In Exp. Abstr., 64th Ann. Internat. Mtg., pages 1306-1309. Soc. of Expl. Geoph.

Cooper, N. (2004). A world of reality - Designing land 3D programs for signal, noise and prestack migration - Part 2. The Leading Edge, 23:1230-1235.

Cox, M. J. G. (1999). Static corrections for seismic reflection surveys. Soc. of Expl. Geophys.

Gersztenkorn, A. and Scales, J. A. (1988). Smoothing seismic tomograms with alpha-trimmed means. Geophysical Journal, 92:67-72.

Herman, G. C., Milligan, P. A., Dong, Q., and Rector, J. W. (2000). Analysis and removal of multiply scattered tube waves. Geophysics, 65(3):745-754.

Hindriks, C. O. H. and Verschuur, D. J. (2001). CFP approach to the complex near surface. In Expanded abstracts, 71st Ann. Internat. Mtg., pages 1863-1866. Soc. of Expl. Geoph.

Kelamis, P., Erickson, K. E., Verschuur, D. J., and Berkhout, A. J. (2002). Velocity-independent redatuming: A new approach to the near-surface problem in land seismic data processing. The Leading Edge, 21:730 735 .

Levander, A. R. (1990). Seismic scattering near the Earth's surface. Pure Appl. Geophys., 132:21-47.

Mars, J., Rector, J., and Lazaratos, S. K. (1999). Filter formulation and wavefield separation of crosswellseismic data. Geophysical Prospecting, 47:610-636.

McMechan, G. A. and Chen, H. W. (1990). Implicit static corrections in prestack migration of commonsource data. Geophysics, 55:757-760.

Morse, P. F. and Hildebrandt, G. F. (1988). Ground-roll suppression by the stackarray. In Exp. Abstr., 58th Ann. Internat. Mtg., page S14.5. Soc. of Expl. Geoph.

Muyzert, E. and Vermeer, P. (2004). The impact if acquisition perturbations on land seismic data. In Exp. Abstr., 74th Ann. Internat. Mtg., page ACQ 3.5. Soc. of Expl. Geoph.

Neidell, N. S. and Taner, M. T. (1971). Semblance and other coherency measures for multichannel data. Geophysics, 36:482-497. 
Panea, I., Drijkoningen, G. G., Bertotti, G., and Matenco, L. (2003). Effect of perturbation on array forming. In Exp. Abstr., 73rd Ann. Internat. Mtg., pages 1-4. Soc. of Expl. Geoph.

Perkins, C. and Calvert, R. (2001). Mixed phase surface consistent deconvolution without phase unwrapping. In Ext. Abstr., 63th Mtg., pages A-23. Europ. Assn. Geophys. Eng.

Reshef, M. (1991). Depth migration from irregular surfaces with depth extrapolation methods. Geophysics, 56:119-122.

Telford, W. M., Geldart, L. P., and Sheriff, R. E. (1990). Applied Geophysics. Cambridge.

Van den Berg, P. M. (2002). Nonlinear scalar inverse scattering: algorithms and applications. In Pike, R. and Sabatier, P., editors, Scattering and inverse scattering in pure and applied science, pages 142-161. Academic Press.

Yilmaz, O. (2001). Seimic data analysis. Soc. of Expl. Geophys. 